\title{
Determinação de desmineralização óssea em gatos após tirotoxicose experimental
}

Fabiano Séllos COSTA ${ }^{1,3}$

Mauro José Lahm

CARDOSO ${ }^{2,3}$

Lucy Marie Ribeiro MUNIZ ${ }^{3}$

Luiz Carlos VULCANO ${ }^{3}$

Carlos Roberto PADOVANI ${ }^{4}$

\section{Correspondência para:}

FABIANOSÉLLOS COSTA

Departamento de Zootecnia e Economia

Rural

Centro de Ciências Agrárias

Universidade Federal do Espírito Santo

Alto Universitário, $\mathrm{s} / \mathrm{n}$

29500-000 - Alegre-ES

fabianosellos@cca.ufes.br

Recebido para publicação: 28/01/2005 Aprovado para publicação: 28/05/2005

\begin{abstract}
1 - Departamento de Zootecnia e Economia Rural do Centro de Ciências Agrárias da Universidade Federal do Espírito Santo, Alegre - ES

2 - Curso de Medicina Veterinária da Faculdade Luiz Meneghel, Bandeirantes - PR

3 - Departamento de Reprodução Animal e Radiologia Veterinária da Faculdade de Medicina Veterinária e Zootecnia da Universidade Estadual Paulista, Botucatu - SP 4 - Departamento de Bioestatística da Faculdade de Medicina Veterinária e Zootecnia da Universidade Estadual Paulista, Botucatu - SP
\end{abstract}

\section{Resumo}

O hipertiroidismo é capaz de proporcionar efeitos sobre o metabolismo ósseo tanto em humanos como em animais. Para melhor avaliar este fato em gatos, em 16 animais foram induzidos a tirotoxicose a partir da administração oral de $150 \mu \mathrm{g} / \mathrm{kg}$ de levotiroxina sódica a cada 24 horas durante 42 dias. Os níveis hormonais foram avaliados por radioimunoensaio e a densidade mineral óssea da extremidade distal do rádio direito foi mensurada através de densitometria óptica radiográfica. Foi possível observar, a partir da primeira semana de experimento, significativa elevação sérica de T4 livre e T4 total acompanhada de desmineralização óssea do rádio.

\section{Introdução}

O hipertiroidismo felino é uma alteração clínica multissistêmica resultante das excessivas concentrações dos hormônios tiroidianos (tirotoxicose), sendo atualmente considerado o distúrbio endócrino de maior incidência nos felinos domésticos ${ }^{1}$. As alterações clínicas em gatos com esta enfermidade são variáveis e irão depender diretamente do estágio em que a doença se encontra e da coexistência de outras alterações sistêmicas ${ }^{2,3,4}$.

Em humanos, o hipertiroidismo é conhecido como um distúrbio capaz de promover osteoporose como conseqüência do aumento da taxa de reabsorção óssea ${ }^{5,6}$. Desta forma, a ocorrência da doença é considerada como um fator de risco para desmineralização óssea, podendo estar correlacionada com o diagnóstico de fraturas patológicas $5,7,8,9,10,11,12,13,14$.

A desmineralização que ocorre é decorrente de uma estimulação global do remodelamento do tecido ósseo, acelerando a perda óssea trabecular e cortical ${ }^{11}$. Achados histológicos de tecido ósseo em pacientes humanos com hipertiroidismo tem sido caracterizados com um padrão histomorfométrico onde se evidencia tanto de um aumento da atividade osteoblástica e como da atividade osteoclástica ${ }^{15}$.

Nos animais, os hormônios tiroidianos são capazes de desencadear graves efeitos sobre o metabolismo ósseo ${ }^{16,17,18,19}$, incluindo um aumento na velocidade de formação e de reabsorção óssea e alterações nos níveis plasmáticos de cálcio e fósforo ${ }^{19}$. Em felinos, apesar da escassez de informações sobre este ponto, é citado que, nesta espécie, é possível ocorrer uma perda de massa óssea em decorrência do hipertiroidismo, podendo ocorrer fraturas patológicas nos ossos longos e corpos vertebrais dos animais acometidos ${ }^{19,20}$.

A reposição hormonal em excesso em humanos com hipotiroidismo, principalmente após a menopausa nas mulheres, pode produzir um estado hipermetabólico idêntico ao observado na tirotoxicose endógena, podendo ter como seqüela um remodelamento ósseo exagera- 
$\mathrm{do}^{5,7,8}$. Este fato assume destacada importância, uma vez que os hormônios da tiróide estão entre as medicações mais freqüentemente prescritas na medicina humana e a administração de uma dose excedida pode ocasionalmente ser encontrada ${ }^{7}$.

A determinação da densidade mineral óssea é um fator fundamental para a definição do quadro de desmineralização, servindo também para o estabelecimento de um protocolo terapêutico e o monitoramento dos pacientes acometidos, podendo desta forma prevenir a ocorrência de fraturas patológicas ${ }^{13}$. Diferentes técnicas densitométricas são descritas na medicina veterinária e humana, visando proporcionar precisão nos dados e boas condições de trabalho. Inúmeros relatos demonstram que a técnica de densitometria óptica em imagens radiográficas é útil para avaliação da densidade mineral óssea, pois apresenta-se com alta precisão, tendo o seu uso uma importância acentuada ${ }^{21,22,23,24,25,26}$.

Objetivou-se neste trabalho induzir gatos normais ao estado de tirotoxicose durante um intervalo de tempo e, a partir da elevação dos níveis hormonais, pretendeu-se identificar possíveis alterações na densidade mineral óssea. Em complementação, objetivou-se correlacionar, nos diferentes momentos experimentais, os níveis séricos dos hormônios tiroidianos com os valores densitométricos.

\section{Materiais e Métodos}

Foram utilizados 16 gatos domésticos (Felis catus, L. 1758), adultos, sem distinção de sexo ou raça e sem alterações clínicas ou laboratoriais que pudessem interferir na realização da pesquisa. Animais em fase de crescimento, castrados e fêmeas gestantes foram excluídos do grupo experimental. Após a seleção, os animais foram acondicinados em um gatil com acesso ao sol, sendo administrada alimentação (ração seca) e água à vontade. Após a realização do experimento, os gatos passaram por um período de desintoxicação de 10 dias, sendo posteriormente doados.
Para indução ao estado de tirotoxicose, administrou-se por via oral comprimidos de levotiroxina sódica a cada 24 horas durante 42 dias, na dose de 150 $\mu \mathrm{g} / \mathrm{kg}$. Semanalmente os gatos eram pesados e as doses para cada animal eram recalculadas conforme a necessidade. Todos os dados experimentais foram coletados imediata-mente antes do início da administração do medicamento, sendo realizadas posterior-mente avaliações a cada sete dias até o término do período experimental.

A dosagem dos níveis séricos dos hormônios tiroidianos (T3 total, T4 livre e T4 total) foram obtidas em todos os momentos, pela técnica de radioimunoensaio em fase sólida, utilizando-se conjunto de reagente comercial sem qualquer tipo de extração química ou processo de purificação, tendo como elemento radioativo traçador o $\operatorname{Iodo}^{125}$.

A avaliação da densidade mineral óssea foi realizada pela técnica de densitometria óptica em imagens radiográficas, conforme técnica já previamente descrita na literatura ${ }^{21,22,23,24,25,26}$. A área avaliada foi a extremidade distal do rádio direito e, para melhor análise seqüencial dos valores densitométricos, padronizou-se a seleção da região da metáfise localizada $5 \mathrm{~mm}$ acima da cicatriz epifisária (Figura 1) em todos os animais e em todos os momentos experimentais, sendo posteriormente feita a mensuração da densidade mineral óssea (Figura 2).

A análise estatística das variáveis ao longo dos diferentes momentos foi realizada considerando-se a técnica de análise de variância para medidas repetidas. Quando a variável apresentou as características de distribuição normal de probabilidade, o procedimento foi paramétrico. $\mathrm{Na}$ ausência de características de distribuição normal de probabilidade utilizou-se o procedimento não-paramétrico. Para a interpretação das letras deve-se considerar que duas médias ou medianas seguidas pelo menos de uma mesma letra não diferem ao nível de $5 \%$ de 
significância. Para o estudo da correlação entre as variáveis em cada um dos momentos, utilizou-se o coeficiente de correlação linear de Pearson.

\section{Resultados e Discussão}

O protocolo de indução ao hipertiroismo utilizado foi eficaz, ocorrendo por uma elevação estatisticamente significativa dos níveis séricos de $\mathrm{T}_{4}$ livre (Tabela 1) e $\mathrm{T}_{4}$ total (Tabela 2) Esse aumento esteve presente a partir da primeira semana de administração oral de levotiroxina sódica, mantendo-se os altos níveis hormonais até o término do período experimental de 42 dias. Os níveis plasmáticos de $\mathrm{T}_{3}$ total não apresentaram alterações significativas (Tabela 3). Dessa forma, a dosagem do $T_{3}$ total não esteve associada com as alterações dos níveis de $\mathrm{T}_{4}$ livre e $\mathrm{T}_{4}$ total dos gatos ou com as alterações presentes no metabolismo ósseo.

Assim, como em nossa pesquisa, outros autores conseguiram promover uma indução experimental à tirotoxicose a partir da administração oral de levotiroxina sódica ${ }^{16,17,18}$. São descritos trabalhos em ratos $^{17,18}$ e cães ${ }^{16}$, em que foi possível a demonstração de alterações do metabolismo do tecido ósseo.

Foi caracterizada uma queda, estatisticamente significativa, da densidade mineral óssea da extremidade distal do rádio dos animais submetidos ao protocolo experimental de indução à tirotoxicose já na primeira semana de tratamento com levotiroxina sódica. Até o término do período experimental ocorreu um gradual decréscimo dos valores médios da densidade mineral óssea, mantendo-se cada vez menores os valores densitométricos (Tabela 4). Alterações desta natureza já foram amplamente descritas na medicina humana ${ }^{5,6,7,9,13,14,15,16,27}$, entretanto, poucos autores relatam este fato em animais 1,8,17,27.

Em todos os momentos, a partir do início da administração hormonal, foi observada uma correlação negativa entre a densidade mineral óssea da extremidade distal do rádio e os níveis plasmáticos de $\mathrm{T}_{4}$ livre e $\mathrm{T}_{4}$ total; entretanto, este fato não foi verificado com os níveis de $\mathrm{T}_{3}$ total. Uma correlação estatisticamente significativa $(\mathrm{p}<0,05)$ só esteve presente após duas semanas de tratamento hormonal, onde foi constatada uma forte correlação negativa entre os níveis de $\mathrm{T}_{4}$ total e os valores densitométricos (Tabela 5).

A partir de radiografias convencionais em humanos, a visualização de manifestações ósseas nos casos de tirotoxicose endógena é de rara ocorrência ${ }^{9}$ Nesta pesquisa, este acontecimento também foi verificado na tirotoxicose experimental. Radiograficamente não foi possível estabelecer o diagnóstico de perda de massa óssea nos gatos estudados a partir de radiografias simples. Este fato reforça a importância da avaliação densitométrica para um diagnóstico precoce das lesões ósseas decorrentes do hipertiroidismo.

As semelhanças nas alterações no tecido ósseo na tirotoxicose de origem exógena e endógena em humanos, conforme descrito em mulheres submetidas a uma reposição hormonal excessiva ${ }^{17}$, nos levam a considerar que fato semelhante possa ocorrer nos gatos domésticos. Dessa forma, possivelmente a perda de massa óssea, observada nos gatos do presente estudo, também ocorrerá de forma semelhante no hipertiroidismo felino de origem endógena. Outro fato relevante, concordando com os relatos de Fallon et al. ${ }^{7}$, é com relação à prescrição de hormônios tiroidianos. Nossos resultados demonstram que, possíveis erros no cálculo da dose dos medicamentos para reposição hormonal nos gatos, levando à administração de uma dosagem excessiva, podem proporcionar efeitos no metabolismo ósseo dos animais.

Kung e $\mathrm{Ng}^{17}$ demonstram significativa diminuição da densidade mineral óssea do fêmur, da coluna e da cauda de ratos induzidos à tirotoxicose após avaliação densitométrica. As alterações de densidade mineral óssea já estavam presentes com três semanas após o início de administração 
hormonal, o que concorda com nossos dados, segundo os quais, um curto período de tirotoxicose exógena pode afetar o metabolismo ósseo.

A técnica de densitometria óptica em imagens radiográficas, demonstrou ser uma útil e de fácil realização. Foi possível uma caracterização precisa do quadro de perda de massa óssea, assim como um acompanhamento periódico das alterações da densidade mineral óssea. Outros autores também já verificaram a sua aplicabilidade e precisão em cães ${ }^{25}$, eqüinos ${ }^{26}$, gatos domésticos ${ }^{24} \mathrm{e}$ em peças ósseas de frangos ${ }^{23}$.

Durante o período experimental, não foram verificadas fraturas patológicas nos animais induzidos ao estado de tirotoxicose. Em humanos, é citado que os efeitos dos hormônios tiroidianos proporcionam uma maior fragilidade do osso. Assim, o hipertiroidismo, ou uma história prévia da doença, aumenta o risco de fraturas patológicas decorrentes da osteoporose $e^{5,8,10,13,14}$. Nos gatos utilizados nesta pesquisa, esse fator complicante não ocorreu durante o período experimental.

Tabela 1 - Medidas descritivas do $\mathrm{T}_{3}$ total (ng/dL) e resultado do teste estatístico da comparação entre os momentos. Botucatu, 2002

\begin{tabular}{cccccccc}
\hline Medidas descritivas & \multicolumn{7}{c}{ Momentos } \\
& M0 & M1 & M2 & M3 & M4 & M5 & M6 \\
\cline { 2 - 8 } & & & & & & \\
Valor mínimo & 27,08 & 32,71 & 23,89 & 19,25 & 6,39 & 21,65 & 28,99 \\
Primeiro quartil & 52,20 & 46,30 & 55,25 & 46,92 & 35,35 & 46,20 & 53,64 \\
Mediana & 60,03 a & 64,61 a & 75,79 a & 66,80 a & 66,86 a & 53,95 a & 66,95 a \\
Terceiro quartil & 75,57 & 120,58 & 94,45 & 81,73 & 90,46 & 92,34 & 82,86 \\
Valor máximo & 98,59 & 187,37 & 135,33 & 138,28 & 103,60 & 126,87 & 138,3 \\
Média & 64,69 & 85,01 & 77,76 & 69,11 & 63,23 & 68,95 & 70,46 \\
Desvio padrão & 17,95 & 49,63 & 31,97 & 34,12 & 31,93 & 33,77 & 29,19 \\
Resultado do teste & & \multicolumn{7}{c}{$4,58(\mathrm{P}>0,05)$} & & \\
estatístico & & 7 &
\end{tabular}

Tabela 2 - Medidas descritivas do T 4 livre (ng/dl) e resultado do teste estatístico da comparação entre os momentos. Botucatu, 2002

\begin{tabular}{cccccccc}
\hline Medidas descritivas & \multicolumn{7}{c}{ Momentos } \\
\hline & M0 & $M 1$ & $M 2$ & $M 3$ & $M 4$ & $M 5$ & M6 \\
\cline { 2 - 8 } & & & & & & & \\
Valor mínimo & 0,12 & 0,22 & 0,09 & 0,05 & 0,14 & 0,19 & 0,30 \\
Primeiro quartil & 0,22 & 0,52 & 0,71 & 0,62 & 0,82 & 0,46 & 0,55 \\
Mediana & $0,31 \mathrm{a}$ & $0,79 \mathrm{~b}$ & $1,09 \mathrm{~b}$ & $0,93 \mathrm{~b}$ & $1,09 \mathrm{~b}$ & $1,04 \mathrm{~b}$ & $0,71 \mathrm{~b}$ \\
Terceiro quartil & 0,53 & 0,79 & 1,09 & 0,93 & 1,09 & 1,04 & 0,71 \\
Valor máximo & 0,89 & 2,74 & 2,47 & 2,29 & 4,11 & 2,21 & 2,02 \\
Média & 0,39 & 1,16 & 1,11 & 0,99 & 1,26 & 1,11 & 0,88 \\
Desvio padrão & 0,23 & 0,86 & 0,65 & 0,61 & 0,92 & 0,67 & 0,48 \\
Resultado do teste & & & & & & &
\end{tabular}


Tabela 3 - Medidas descritivas do $\mathrm{T}_{4}$ total $(\mathrm{mg} / \mathrm{dl})$ e resultado do teste estatístico da comparação entre os momentos. Botucatu, 2002

\begin{tabular}{cccccccc}
\hline Medidas descritivas & \multicolumn{7}{c}{ Momentos } \\
\hline & M0 & M1 & M2 & M3 & M4 & M5 & M6 \\
\cline { 2 - 8 } & & & & & & & \\
Valor mínimo & 0,80 & 2,01 & 1,14 & 1,75 & 1,76 & 1,31 & 1,90 \\
Primeiro quartil & 1,85 & 4,37 & 3,94 & 4,26 & 5,09 & 5,80 & 4,58 \\
Mediana & $2,43 \mathrm{a}$ & $6,09 \mathrm{~b}$ & $6,27 \mathrm{~b}$ & $8,27 \mathrm{~b}$ & $7,92 \mathrm{~b}$ & $9,79 \mathrm{~b}$ & $7,16 \mathrm{~b}$ \\
Terceiro quartil & 3,98 & 10,20 & 10,09 & 12,15 & 11,11 & 12,40 & 8,08 \\
Valor máximo & 6,31 & 16,41 & 14,64 & 16,36 & 18,78 & 14,90 & 15,40 \\
Média & 2,96 & 7,45 & 6,93 & 8,53 & 8,20 & 8,98 & 7,06 \\
Desvio padrão & 1,71 & 4,27 & 3,98 & 4,82 & 4,76 & 4,40 & 3,79 \\
Resultado do teste & & \multicolumn{7}{c}{$22,74(\mathrm{P}<0,001)$} & & \\
estatístico & & 70 &
\end{tabular}

Tabela 4-Medidas descritivas da densidade mineral óssea (mmAl) e resultado do teste estatístico da comparação entre os momentos. Botucatu, 2002

\begin{tabular}{|c|c|c|c|c|c|c|c|}
\hline \multirow[t]{2}{*}{ Medidas descritivas } & \multicolumn{7}{|c|}{ Momentos } \\
\hline & MO & M1 & M2 & M3 & M4 & M5 & M6 \\
\hline Valor mínimo & 1,03 & 0,92 & 0,91 & 0,92 & 0,90 & 1,00 & 0,90 \\
\hline Primeiro quartil & 1,28 & 1,27 & 1,25 & 1,26 & 1,22 & 1,22 & 1,26 \\
\hline Mediana & 1,47 & 1,41 & 1,41 & 1,36 & 1,37 & 1,37 & 1,36 \\
\hline Terceiro quartil & 1,57 & 1,56 & 1,56 & 1,50 & 1,51 & 1,48 & 1,46 \\
\hline Valor máximo & 1,78 & 1,69 & 1,69 & 1,74 & 1,67 & 1,74 & 1,74 \\
\hline Média & $1,43 b$ & $1,38 \mathrm{a}$ & 1,38 a & $1,35 \mathrm{a}$ & $1,35 \mathrm{a}$ & $1,34 \mathrm{a}$ & $1,33 \mathrm{a}$ \\
\hline Desvio padrão & 0,23 & 0,21 & 0,22 & 0,2 & 0,21 & 0,20 & 0,21 \\
\hline $\begin{array}{c}\text { Resultado do teste } \\
\text { estatístico }\end{array}$ & \multicolumn{7}{|c|}{$6,11(\mathrm{P}<0,001)$} \\
\hline
\end{tabular}

Tabela 5 - Medidas de associação entre as variáveis estudadas nos diferentes momentos experimentais. Botucatu, 2002.

\begin{tabular}{cccccccc}
\hline $\begin{array}{c}\text { Associação entre as } \\
\text { variáveis }\end{array}$ & M0 & M1 & M2 & M3 & M4 & M5 & M6 \\
\hline DMO x T3 total & $-0,30$ & 0,23 & $-0,06$ & $-0,07$ & $-0,10$ & $-0,20$ & $-0,14$ \\
DMO x T4 livre & $-0,26$ & $-0,15$ & $-0,15$ & $-0,15$ & $-0,12$ & $-0,27$ & $-0,36$ \\
DMO x T4 total & $-0,24$ & $-0,27$ & $-0,51 *$ & $-0,24$ & $-0,08$ & $-0,19$ & $-0,37$ \\
\hline
\end{tabular}




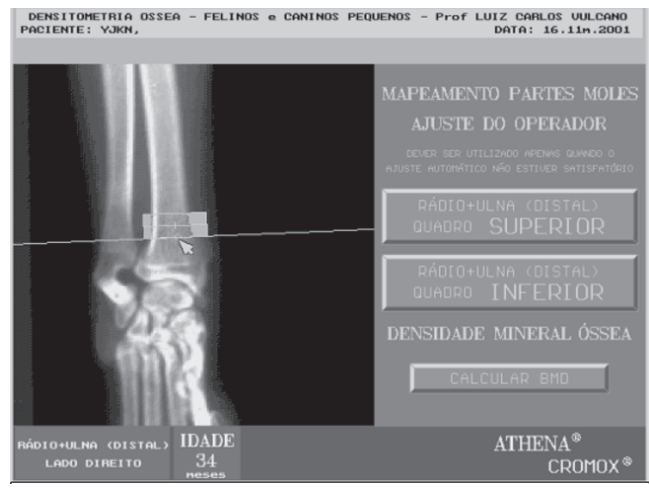

Figura 1 - Delimitação da região de tecido ósseo a ser realizada a análise densitométrica

\section{Conclusões}

O protocolo de indução a tirotoxicose utilizado neste experimento é eficaz e aumenta significativamente os níveis séricos de T4 livre e T4 total nos felinos domésticos a partir da primeira semana de tratamento. Essa elevação dos hormônios tiroidianos foi responsável por uma diminuição estatisticamente significante da

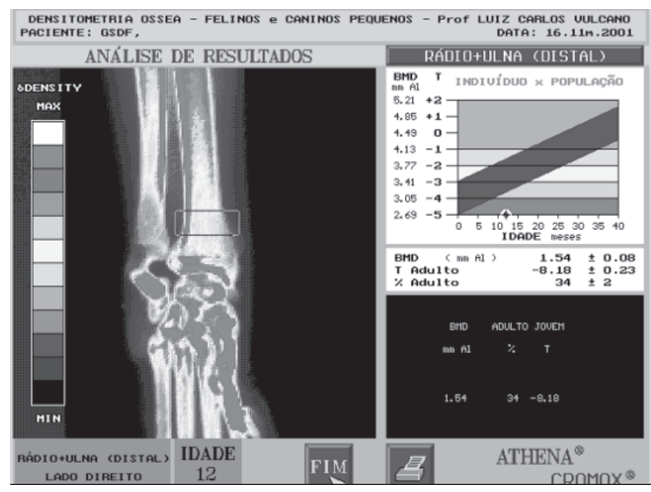

Figura 2 - Cálculo da densidade mineral óssea da área selecionada da extremidade distal do rádio

densidade mineral óssea da extremidade distal do rádio direito dos gatos também a partir da primeira semana do período experimental. Apesar da significativa desmineralização óssea, não ocorreram fraturas patológicas. A densitometria óptica em imagens radiográficas é uma metodologia útil, precisa e que proporciona uma adequada avaliação seqüencial da densidade mineral óssea.

\section{Determination of bone demineralization in cats after experimental thyrotoxicosis}

\begin{abstract}
Hyperthyroidism can result in serious effects on the bone metabolism in humans as well as animals. For a better characterization of thyrotoxicosis effects, 16 cats were induced into thyrotoxicosis by intaking a dose of $150 \mu \mathrm{g} / \mathrm{kg}$ of sodium L-thyroxine every 24 hours during 42 days. The hormonal levels were evaluated by radioimmunoassay technique and the bone mineral density of the right distal radius extremity was measured through the radiographic optical densitometry. Was verified significant bone demineralization seven days of hormonal intake as weel as radius demineralization.
\end{abstract}

Key-words: Hyperthyroidism. Thyrotoxicosis. Cat. Bone demineralization.

\section{Referências}

1 PETERSON, M. E. et al. Feline hyperthyroidism: pretreatment clinical and laboratory evaluation of 131 cases. J.A.V.M.A., v. 183, n. 1, p. 103-110, 1983.

2 BROUSSARD, J. D: PETERSON, M. E: FOX, P. R. Changes in clinical and laboratory findings in cats with hyperthyroidism from 1983 to 1993. J.A.V.M.A., v. 206, n. 302, 1995.

3 PETERSON, M. E. Hyperthyroid diseases. In:
ETTINGER, S. J.: FELDMAN, E. C. Textbook of veterinary internal medicine. Philadephia: WB Saunders, 1995. p.1466-1501.

4 THODAY, K. L.; MOONEY, C. T. Historical, clinical and laboratory features of 126 hyperthyroid cats. Veterinary Records, v.131, p. 257-264.

5 JODAR, E. et al. Bone loss in hyperthyroid patients and in former hyperthyroid patients controlled on medical therapy: influence of aetiology and menopause. Clinical Endocrinology, v. 47, p. 279-285, 1997. 6 MOSEKILDE, L.: ERIKSEN, E. F., CHARLES, P. Effects 
of thyroid hormones on bone and mineral metabolism. Endocrinology and Metabolism Clinics of North America, v. 19, n. 1, p. 35-63, 1990.

7 FALLON M. D. et al. Exogenous hyperthyroidism with osteoporosis. Arch. Intern. Med., v. 143, p. 442444, 1983.

8 FOLDES, J. et al. Bone mineral density in patients with endogenous subclinical hyperthyroidism: is this thyroid status a risk factor for osteoporosis? Clinical Endocrinol, v. 39, p. 521-527, 1993.

9 KOUTRAS, D. A. et al. Radiological signs of bone loss in hyperthyroidism. Br. J. Radiol., v. 46, p. 695698, 1973.

10 LÉGER, J. et al. Ostéopénie grave chez de jeunes enfants atteints d'hyperthyroidie. Arch. Fr. Pediatr., v. 43, p.123-125, 1986.

11 MEUNIER, P. J. Hyperthyroidies et ostéoporose. Annales d'Endocrinologie, v. 56, p. 57-59, 1995.

12 MUNDY, G. R. et al. Direct stimulation of bone resorption by thyroid hormones. J. Clin. Invet., v. 58, p. 529-534, 1976.

13 SCHARLA, S. H. et al. Prevalence of low bone mass and endocrine disorders in hip fracture patients in Sourthern Germany. Exp. Clin. Endocrinol. Diabetes, v.107, p.547-554, 1999.

14 SEEGER, L. L. Bone density determination. Spine, v. 22, n. 245, p 49s-57s, 1997.

15 ALLAIN, T. J., MCGREGOR, A. M. Thyroid hormones and bone. J. Endocrinol.; v. 139, p. 9-18, 1993.

16 ADAMS, P: JOWSEY, J. Bone and mineral metabolism in hyperthyroidism: an experimental study. Endocrinol: v. 81, p. 735-740, 1966.

17 KUNG, A. W: NG, F. A rat model of thyroid hormone-induced bone loss: effect of antiresorptive agents on regional bone density and osteocalcin gene expression. Thyroid, v.4, n.1, p.93-98, 1994.

18 SERAKIDES, R. Relação tireóide-gônadas e níveis plasmáticos de fósforo cálcio e fosfatase alcalina em ratas. Arq. Bras. Med. Vet. Zootec., v. 52, n. 6, p. 57985, 2000 .

20 NEWTON, C. D.: BIERY, D. N. Moléstias esqueléticas. In: ETTINGER, S. J. Tratado de medicina interna veterinária. São Paulo: Manole, 1992, v. 4, p. 2494-2517.

21 LOUZADA, M. J. Q. Otimização da técnica de densitometria óptica em imagens radiográficas em peças ósseas - estudo "in vitro". 1994. 191 p. Tese (Doutorado) - Faculdade de Engenharia Elétrica, Universidade Estadual de Campinas, Campinas, 1994.

22 LOUZADA, M. J. Q. et al. Avaliações da densidade óssea em radiografias - I metodologia. In: CONGRESSO BRASILEIRO DE ORTOPEDIA E TRAUMATOLOGIA,

\section{0, 1996, Curitiba. Anais.}

23 LOUZADA, M. J. Q. et al. Densidade de peças ósseas de frangos. Estudo pela densitometria óptica radiográfica. Vet e Zootec., v. 9, p. 95-109, p1997.

24 RAHAL, S. C. et al. Emprego da densitometria óptica radiográfica na avaliação do hiperparatireoidismo secundário nutricional induzido em gatos jovens. In: CONGRESSO INTERNACIONAL DE MEDICINA FELINA, 2001, Rio de Janeiro. Anais. Rio de Janeiro, 2001. p. 35

25 VULCANO, L. C. et al. Determination of the normal values of density of the radius in Rottweilers, using radiographic otical densitometry (experimental study). In: CONGRESSO MUNDIAL DE MEDICINA VETERINÁRIA DE PEQUENOS ANIMAIS, 1998, Buenos Aires. Anais.

26 VULCANO, L. C. Determinação e padronização dos valores normais da densidade mineral óssea (DMO) do carpo acessório de eqüinos em crescimento da raça Puro Sangue Inglês (PSI) por meio da densitometria óptica radiográfica. 2001. 52 p. Tese (Livre Docência) - Faculdade de Medicina Veterinária e Zootecnia, Universidade Estadual Paulista, 19 WASSERMAN, R. H. Ossos In: SWENSON, M.J. Dukes fisiologia dos animais domésticos. Rio de Janeiro: Guanabara Koogan, 1988., 10. ed. p. 413-427.

27 MELSEN, F.: MOSEKILDE, L. Morphometric and dynamic studies of bone changes in hyperthyroidism. Acta Pathol. Microbiol. Scand., v. 85, n. 141, 1977. 